\title{
Primary Care Physician Communication at Hospital Discharge Reduces Medication Discrepancies
}

\author{
Lee A. Lindquist, MD, MPH, MBA*, Atsuko Yamahiro, MD, Arianne Garrett, BS, Charles Zei, BS, Joseph M. Feinglass, PhD, MPH
}

Division of General Internal Medicine and Geriatrics, Northwestern University Feinberg School of Medicine, Chicago, Illinois.

BACKGROUND: Medication discrepancies are common as patients transition from hospital to home. Errors with postdischarge medication regimens may play a role in hospital readmissions.

OBJECTIVES: To determine whether primary care physician (PCP) contact with patients at hospital discharge impacts the frequency of medication discrepancies at 24 hours post-discharge.

DESIGN: With the PCP-Enhanced Discharge Communication Intervention, PCPs were asked to speak with treating hospitalists and contact patients within 24 hours of hospital discharge (either in person or by phone) to discuss any hospital medication changes. Research staff enrolled subjects during their hospitalization and telephoned subjects 48 hours post-discharge to determine medication discrepancies and PCP contact.

PARTICIPANTS: One hundred fourteen communitydwelling adults, admitted to acute medicine services $>24$ hours on $\geq 5$ medications.
RESULTS: Of the 114 subjects enrolled in the hospital, 75 subjects completed 48 hours postdischarge phone interviews. Of the 75 study patients, 39 patients (50.6\%) experienced a total of 84 medication discrepancies (mean, 2.1 discrepancies/patient). Subjects who were contacted by their PCP at discharge were $70 \%$ less likely to have a discrepancy when compared with those not contacted $(P=0.04)$. Males were 4.34 times more likely to have a discrepancy $(P=0.02)$.

CONCLUSION: PCP communication with patients within 24 hours of discharge was associated with decreased medication discrepancies. Our results further demonstrate the importance of PCP involvement in the hospital discharge process. Journal of Hospital Medicine 2013;8:672-677. (C) 2013 Society of Hospital Medicine
Medication errors occur frequently when patients transition care between providers, such as the transition from hospital to home. ${ }^{1}$ Approximately $50 \%$ of about 3 million adults per year over 65 years of age transitioning from hospital to home experience a medication discrepancy. ${ }^{1}$ At hospital discharge, patients with complex medical problems are prescribed multiple medications with complex dosing and schedules. To add to this complexity, many patients also have cognitive impairment, variable health literacy, and limited social and financial support. Patients with medication discrepancies have significantly higher rates of rehospitalization compared to those without medication discrepancies. ${ }^{2}$ Thus, interventions that focus on patients at greater risk of medication discrepancies may identify those at greater risk of subsequent rehospitalization and potentially reduce the rates of readmissions. ${ }^{3}$

\footnotetext{
*Address for correspondence and reprint requests: Lee Lindquist, MD, Associate Professor of Medicine, Northwestern University Feinberg School of Medicine, 750 North Lake Shore Drive, 10th Floor, Chicago, IL 60611; Telephone: 312-695-4525; Fax: 312-695-6060; E-mail: lal425@md. northwestern.edu
}

Additional Supporting Information may be found in the online version of this article.

Received: June 28, 2013; Revised: September 27, 2013; Accepted: September 27, 2013

2013 Society of Hospital Medicine DOI 10.1002/jhm.2098

Published online in Wiley Online Library (Wileyonlinelibrary.com).
There is limited evidence to date on the effectiveness of interventions to decrease post-hospitalization medication discrepancies. ${ }^{3-6}$ Previous transitional care interventions have been expensive and difficult to sustain due to the need for multiple additional transitional care personnel (e.g., care managers, nurses, pharmacists, transitional care coaches)..$^{7-10}$ Moreover, these additional personnel may further fragment the care of hospitalized patients with additional handoffs.

As both hospitalists and outpatient primary care physicians (PCPs) are being expected to care for larger numbers of increasingly sicker patients, the communication handoff of patient information at discharge remains a challenge. ${ }^{11}$ Both patients and PCPs often obtain incomplete or inaccurate information and instructions at discharge. Follow-up appointments are often missed or delayed. ${ }^{12}$ Many hospital-based interventions do not directly involve the PCP or take advantage of the already established PCP-patient relationship.

We conducted a pilot study examining whether enhancing PCP communication with patients at hospital discharge would impact medication discrepancies and improve the safety of the patient as they transitioned home from the hospital.

\section{METHODS}

\section{Recruitment of Subjects}

The institutional review board of Northwestern University approved this study. Accessing the hospital 
electronic health record, research staff queried admission information and contacted the hospital physician to determine potential subject eligibility. Research staff recruited consecutive community-dwelling adults aged 18 years and older who were hospitalized to the acute medicine services at Northwestern Memorial Hospital and being discharged to their home. The patients recruited had PCPs from practices in the Research and Education for Academic Achievement (REACH) Practice-Based Research Network. The REACH Practice-Based Research Network consists of 8 academic, private, and community-based provider groups affiliated with Northwestern Memorial Hospital and the Northwestern University Feinberg School of Medicine. Subjects were excluded if they (1) were unable to consent to their own procedures while hospitalized, (2) were severely vision impaired that could not be corrected with glasses (because they would be administered tests requiring adequate vision), (3) were reliant on a caregiver or home aide services 8 hours or greater per day, (4) were enrolled in hospice, (5) spoke a language other than English or Spanish, (6) were expected to have a hospital length of stay of $<24$ hours, or (7) were on $<5$ outpatient medications prior to hospitalization.

\section{Initial Patient Interview-Hospital}

Research staff conducted structured in-person surveys of eligible hospitalized subjects in the private hospital rooms of subjects to maintain confidentiality. Subjects received \$20 compensation for their participation. After written informed consent was obtained, research staff obtained demographic information from the subject as well as ascertained their availability in 48 hours, support system, medications, and PCP. Research staff then administered the Short Test of Functional Health Literacy in Adults (sTOFHLA) to determine health literacy. ${ }^{13}$ All research staff received the same training on administering the cognitive testing. The survey lasted between 20 and 30 minutes. At hospital discharge, the medication list was obtained from the discharge instructions listed in the patient's electronic medical record.

\section{The PCP-Enhanced Discharge Communication Intervention}

Research staff and the principal investigator met with hospitalists and REACH outpatient physicians in groups to inform them of the study prior to beginning. When the patient was nearing discharge from the hospital, hospitalists were asked to phone the PCP to discuss the patient's discharge plan and facilitate clinical handoffs to the outpatient setting. Research staff paged the hospitalists with the PCP's contact information and reminded them to contact the PCP. Following the hospitalist-PCP phone call, the PCP contacted the patient within 24 hours of discharge, either in person while in the hospital or by phone at home. The PCP confirmed medications and clarified any posthospital confusion. The contact flexibility (phone or by person and within 24 hours) was planned as PCPs had other responsibilities that would not allow them to be present at the actual discharge. Physicians were asked to review medications and follow-up plans, but phone conversations were not recorded. PCPs were given a laminated card that contained points to discuss on discharge (Figure 1). PCPs were compensated with a $\$ 5$ coffee shop gift card for their time to call patients. This intervention did not involve any additional healthcare personnel.

\section{Postdischarge Phone Interview}

During the initial interview, research staff set up a time to contact the subject by phone at 48 hours postdischarge. Research staff contacted the subject at the scheduled time and attempted contact 3 times within 24 hours of scheduled time. During the phone interview, subjects were asked whether they had contact with their PCP to discuss hospital discharge instructions (if so, when) and how satisfied they were with the contact. The subject was also asked whether they were confused with any aspect of the discharge, and if they were, whether the PCP alleviated the confusion. The subjects were asked whether anyone has assisted with their medications, whether they had medication changes, and which medications they were currently taking in the outpatient setting. Both prescription and over-the-counter medications were included. Over-thecounter medications were included in the study to determine the severity of medication discrepancies if they existed.

The medication list given by the subject was compared with the medical record for the medication list at discharge. Research staff would determine if a discrepancy was present. A discrepancy was considered

1. Why was the patient hospitalized?

2. What medications were changed, added and/or dropped during the hospitalization?

3. Review old medications to be continued

4. When is the follow-up appointment scheduled?

5. What are the remaining test results to follow up on?

FIG. 1. Five key points for PCPs to discuss with patients within 24 hours of discharge. 
(1) omission of a medication prescribed at discharge, (2) addition of a medication that was not prescribed at discharge, (3) different dose, (4) different frequency, or (5) duplication of a medication. If discrepancies existed, the subjects were asked for the reason that the discrepancies may have occurred. If discrepancies were identified, subjects were asked to contact their PCP regarding any medication discrepancies and to clarify any issues about medications they had.

\section{Data Analysis}

Subjects were categorized into whether they were contacted or not contacted by the PCP within 48 hours of discharge from the hospital. Those who were contacted were defined as those who saw a PCP in person at the time of discharge or within 48 hours of discharge or were called within 48 hours. Those who had an appointment with a PCP after 48 hours of discharge, saw a PCP while hospitalized but did not discuss discharge plan, saw a specialist (such as allergist, urologist, wound care specialist), or who spoke to their PCP but did not discuss their discharge plan were categorized as not contacted.

Reasons for medication discrepancies were categorized into patient-associated factors (adverse drug effect, intentional nonadherence, unintentional nonadherence) and system-associated factors (confusion between brand and generic names, discharge instruction incomplete or inaccurate, duplication, incorrect dosage) according to a published medication discrepancy tool. ${ }^{2}$ Discrepancies were categorized as intentional nonadherence if patients knew the regimen but decided not to adhere. On the other hand, unintentional nonadherence was used for discrepancies in which patients were unaware of the regimen and thus the discrepancy. Medication discrepancies were classified as mild, moderate, or severe depending on the medication involved. Mild discrepancies were over-thecounter medications (eg, acetaminophen, laxatives, multivitamins) and topical creams. Severe discrepancies included medications for heart disease (eg, $\beta$-blockers, calcium channel blockers, angiotensin receptor blockers, diuretics), pulmonary disease (eg, inhalers), diabetes (eg, insulin, glyburide), and antibiotics. Moderate discrepancies were those that did not fit the mild or severe categories (eg, prescription pain medication such as narcotics, anxiety medications, bisphosphonates, muscle relaxants).

Statistical analysis was performed with the SPSS 18.0 (SPSS Inc., Chicago, IL). We analyzed data on study patients to estimate the effect of contact with the PCP within 48 hours of discharge on the frequency of any medication discrepancy. We first examined differences between patients who were contacted or not contacted by patient sociodemographic characteristics. $\chi^{2}$ tests were used to analyze the significance of differences in the proportion of medication discrepancies between patients who were contacted and not contacted. Logistic regression analysis was used to test the effect of being contacted on the likelihood of having any prescription medication discrepancy after controlling for patient characteristics (eg, race and ethnicity, age, number of medications, living alone, sex, and TOFHLA score.)

\section{RESULTS}

\section{Sample Characteristics}

Of the 225 patients who met inclusion criteria, 114 subjects were recruited and interviewed by research staff during the hospital stay and 48 hours after discharge. Due to early discharge and staffing reasons, 27 subjects were not able to be approached during discharge. Of the 84 patients who declined the study, the reasons included: not interested in study $(\mathrm{n}=58)$, did not feel well enough to complete or participate $(\mathrm{n}=16)$, did not wish study personnel to have access to personal records $(\mathrm{n}=5)$, and no reason given $(\mathrm{n}=5)$. Of the 114 subjects enrolled in the hospital, 77 subjects completed 48-hour postdischarge phone interviews with research staff. Two patients had missing data, leaving 75 patients who were included in the analysis.

Study patients' age, race and ethnicity, sex, living situation (alone vs not alone), number of medications, mean sTOFHLA score, and medication discrepancy are summarized in Table 1 . Thirty-six percent of

\begin{tabular}{|c|c|c|c|c|}
\hline & All Subjects, $N=77$ & Subject Without PCP Contact, $\mathrm{n}=50$ & Subjects With PCP Contact, $n=27$ & $P$ Value \\
\hline Mean age $\pm S D, y$ & $63.0 \pm 12.2$ & $63.3 \pm 11.9$ & $62.3 \pm 13.1$ & 0.74 \\
\hline Race and ethnicity, n (\%) & & & & 0.35 \\
\hline White/other & $40(53.5)$ & $28(58.3)$ & $12(44.4)$ & \\
\hline Black & $30(40.0)$ & $18(37.5)$ & $12(44.4)$ & \\
\hline Hispanic & $5(6.7)$ & $2(4.2)$ & $3(11.1)$ & \\
\hline Male, $n(\%)$ & $26(34.7)$ & $13(27.1)$ & $13(48.1)$ & 0.06 \\
\hline Lives alone, $n(\%)$ & $30(40.0)$ & $20(41.7)$ & $10(37.0)$ & 0.69 \\
\hline Mean sTOFHLA score \pm SD & $29.6 \pm 7.9$ & $29.4 \pm 7.7$ & $29.9 \pm 7.8$ & 0.75 \\
\hline Mean number of medications & $9.22 \pm 4.9$ & $9.06 \pm 4.7$ & $9.63 \pm 3.5$ & 0.67 \\
\hline Experienced medication discrepancy, n (\%) & $39(52)$ & $28(59.3)$ & $11(40.7)$ & 0.14 \\
\hline
\end{tabular}

NOTE: Abbreviations: PCP, primary care physician; SD, standard deviation; sTOFHLA, Short Form Test of Functional Health Literacy in Adults. 
patients $(\mathrm{n}=27)$ were contacted by the PCP within 48 hours of discharge. Age, living situation (alone vs not alone), number of medications, and mean sTOFHLA score were similar in both groups of contacted versus noncontacted patients. Of those who were contacted, males made up $48.1 \%$ versus $27.1 \%$ for those not contacted $(P=0.06)$. Similarly, $44.4 \%$ of those who were not contacted were black versus $37.5 \%$ among the contacted $(P=0.035)$.

\section{Medication Discrepancies}

Of the 75 study patients, 39 patients $(50.6 \%)$ experienced a total of 84 medication discrepancies. Fiftyeight medication discrepancies were prescription medications, whereas 25 were over-the-counter medications. Of those who had discrepancies, $46.2 \%$ $(\mathrm{n}=18)$ had 1 discrepancy, $23.1 \%(\mathrm{n}=9)$ had 2 discrepancies, $12.8 \%(\mathrm{n}=5)$ had 3 discrepancies, $10.2 \%$ $(n=4)$ had 4 discrepancies, and $7.7 \%(n=3) 5$ or more discrepancies. The mean number of discrepancies per patient was 2.15 per patient. Medication discrepancies were categorized by severity based on the safety profile of the medication involved and type discrepancy (Table 2).

\section{Reasons for Medication Discrepancies}

The subject-provided reasons for medication discrepancies are listed in Table 3 and divided into patient- and system-associated factors. The overall most frequent reason for a discrepancy was the patient's intentional nonadherence. Examples of intentional nonadherence include "not sure of purpose of medication," "did not recognize drug," "did not fill prescription," "did not need prescription," and "wanted to wait longer, so not taking diuretic daily." The second most frequent reason was inaccurate discharge instructions (e.g., discharge instructions with medication changes denoting "no change" but incorrect outpatient thyroid medication dosage listed (Table 3).

\begin{tabular}{lc}
\hline TABLE 2. Medication Discrepancies by Medication \\
Type and Severity $(\mathrm{n}=84)$ \\
\hline & Frequency, $\mathrm{n}(\%)$ \\
\hline Type of medication discrepancy & \\
Over the counter & $26(30.9)$ \\
Prescription medication & $58(69.0)$ \\
Severity of medication discrepancy & \\
Mild $^{*}$ & $28(33.3)$ \\
Moderate $^{\dagger}$ & $24(28.6)$ \\
Severe $^{\dagger}$ & $32(38.1)$
\end{tabular}

\footnotetext{
*Mild discrepancies: discrepancies involving over-the-counter medications (such as Tylenol, laxatives such as Miralax, and multivitamins) and topical creams.

${ }^{\dagger}$ Moderate discrepancies: discrepancies that did not fit the mild or severe categories (prescription pain medication such as narcotics, anxiety medications, bisphosphonates, muscle relaxants).

${ }^{\ddagger}$ Severe discrepancies: discrepancies involving medications for heart disease ( $\beta$-blockers, calcium channel blockers, angiotensin receptor blockers, diuretics), pulmonary disease (inhalers), diabetes (insulin, glyburide), and antibiotics.
}

\begin{tabular}{|c|c|}
\hline Factor & Frequency, $\mathrm{n}(\%)$ \\
\hline \multicolumn{2}{|l|}{ Patient-associated factors } \\
\hline Adverse drug effects & $8(9.5)$ \\
\hline Intentional nonadherence & $50(59.4)$ \\
\hline Unintentional nonadherence & $1(1.2)$ \\
\hline Subtotal & 59 \\
\hline \multicolumn{2}{|l|}{ System-associated factors } \\
\hline Confusion between brand and generic names & $3(3.5)$ \\
\hline Discharge Instructions incomplete or inaccurate & $12(14.2)$ \\
\hline Duplication & $3(3.5)$ \\
\hline Incorrect dosage & $3(3.5)$ \\
\hline Incorrect frequency & $1(1.2)$ \\
\hline Conflicting information from different sources & $3(3.5)$ \\
\hline Subtotal & 25 \\
\hline Total & 84 \\
\hline
\end{tabular}

\section{Logistic Regression Results for the Likelihood of Any Medication Discrepancy}

Logistical regression results are shown in Table 4. Patients who were contacted by their PCP at discharge were $70 \%$ less likely to have a discrepancy when compared with those who were not contacted $(P=0.03)$. This result was controlled for other possible factors including patient sex. Of interest, men were 3.94 times more likely to have a discrepancy when compared with women $(P=0.02)$. There was also a nonsignificant but potentially important association between higher health literacy, measured continuously $(0-X)$ and being more likely to have a discrepancy $(P=0.07)$. Including variables for age, ethnicity, and living alone were nonsignificant and did not change the regression results for contacted patients.

\section{DISCUSSION}

Our results provide evidence that contact with PCPs within 24 hours of hospital discharge can be effective in decreasing medication discrepancies. The PCPEnhanced Discharge Communication Intervention was designed to investigate the value of improving existing lines of communication at discharge without involving any additional healthcare personnel. As a lean discharge intervention, the PCP, the hospitalist, and the

TABLE 4. Logistic Regression Results for the Likelihood of Medication Discrepancy

\begin{tabular}{lcc}
\hline & Odds Ratio & 95\% Confidence Interval \\
\hline Subject contacted by PCP at Discharge & 0.33 & $0.11-0.97$ \\
Male & 3.98 & $1.27-12.49$ \\
Number of medications & 1.09 & $0.96-1.23$ \\
TOHFLA score & 1.09 & $1.01-1.18$ \\
\hline
\end{tabular}

NOTE: Abbreviations: TOHFLA, Test of Functional Health Literacy in Adults. 
patient were the main components to this intervention.

This study was limited in that the sample size was small and that we enrolled consecutive patients. Due to the small sample size, we did not examine hospital readmissions. Further studies are needed to examine whether primary care involvement at discharge would affect hospital readmissions. Another limitation of this study was that the control group was not randomized or preselected. Our study compared those subjects who received a phone call from their PCP to those subjects who did not. Although we instructed PCPs with a standardized script, we did not record or ensure that the phone call-up occurred as such. There is potential variability in how the PCPs conducted their follow-up with patients, and we are unable to measure what was effective and ineffective in reducing medication discrepancies. Another limitation was that the determination of the severity of the medication discrepancy was done by medication involved as opposed to by physician review and adjudication. The study would have been strengthened by interviewing the outpatient physicians on the amount of harm each discrepancy would or did cause the patient.

The most frequent reason for discrepancy was intentional nonadherence. Prior research has shown that intentional nonadherence of medications at hospital discharge is linked to health literacy. ${ }^{14}$ One may postulate that patients with adequate health literacy feel enabled to go against medical advice and chose to not take medications as prescribed.

In our study, patients had the most medication discrepancies in the severe medications category, which involved cardiac, pulmonary, and diabetic medications, compared with the mild and moderate category. This finding may reflect the frequency that these medications are prescribed but are consistent with findings of Coleman et al. ${ }^{2}$ The finding highlights the need to ensure adequate education and understanding of medication regimens for these complex patients. Patients with cardiac, pulmonary, and diabetic disease may benefit from personalized discharge instructions and a more structured and organized medication reconciliation process.

Our study found that males were more likely to have a medication discrepancy than females, which has not been found in previous studies on medication discrepancies. One study on Medicare beneficiaries with congestive heart failure found that men were much more likely to be readmitted than woman within 6 months of discharge. ${ }^{15}$ The reason for the increased risk of medication discrepancy in males is unknown. Gender differences in health have frequently been reported, with men having higher rates of morbidity and mortality than women. ${ }^{16,17}$ The differences are thought to be due to the reluctance of men to seek medical help and consult medical practitioners when needed. It has been known that women use health services more than men, and are more likely than men to report a chronic illness. ${ }^{18}$ When men do present with symptoms, it is often later in the stage of a disease than women and when treatment is less likely to be successful. ${ }^{19}$ It may be that men in this study population had more medication discrepancies as they were reluctant to seek help or ask for clarification regarding medications at discharge.

Of those enrolled, $36 \%$ of patients were contacted by their PCP within 48 hours of discharge. It is unclear if the PCP attempted but was unable to reach the patient or did not attempt to call the patient. Although PCPs were compensated with a $\$ 5$ coffee shop gift card, a larger compensation may insure completion of the patient contact. Further research is needed to determine the reasons why PCPs were not able to complete the phone call.

From a policy standpoint, hospitals that focus solely on hospital-based transition interventions are potentially missing half the problem. The hospital acts as a sender or "pitcher," and the PCP acts as a receiver or "catcher." The receiver needs to be included in the discharge process for a successful patient transition to home. With recent billing changes for transition coding, the Center for Medicare and Medicaid Services recognizes this relationship. ${ }^{20}$ Outpatient PCPs are able to bill for bundled follow-up phone calls and appointments. Instead of paying additional staff to make 48-hour postdischarge phone calls, hospitals should consider partnering with PCPs to ensure a more organized discharge.

Our results showed that PCP communication with patients within 24 hours of discharge was associated with decreased medication discrepancies. The PCP is vital to ensuring a safe transition home from the hospital. Because many patients have an established relationship with their PCP, a bond of trust exists that is often missing with hospital-employed transitional staff. Patients pay attention when a known physician contacts them directly. In our study, patients may have felt comfortable addressing their concerns and questions with their trusted PCP. Subsequently, patients may have been more attuned to the answers their PCP gave and avoided medication errors. Our results further demonstrate the importance of PCP involvement in the hospital discharge process to improve the care of our patients.

\section{References}

1. Forster AJ, Murff HJ, Peterson JF, Gandhi TK, Bates DW. The incidence and severity of adverse events affecting patients after discharge from the hospital. Ann Intern Med. 2003;138(3):161-167.

2. Coleman EA, Smith JD, Raha D, Min SJ. Posthospital medication discrepancies-prevalence and contributing factors. Arch Intern Med. 2005;165(16):1842-1847.

3. Naylor MD, Brooten D, Campbell R, et al. Comprehensive discharge planning for the hospitalized elderly. A randomized clinical trial. Ann Intern Med. 1994;120(12):999-1006.

4. Parry C, Min SJ, Chugh A, Chalmers S, Coleman EA. Further application of the care transitions intervention: results of a randomized controlled trial conducted in a fee-for-service setting. Home Health Care Serv Q. 2009;28(2-3):84-99. 
5. Weinberger M, Oddone EZ, Henderson WG. Does increased access to primary care reduce hospital readmissions? Veterans Affairs Cooperative Study Group on Primary Care and Hospital Readmission. N Engl J Med. 1996;334(22):1441-1446.

6. Koehler BE, Richter KM, Youngblood L, et al. Reduction of 30-day postdischarge hospital readmission or emergency department (ED) visit rates in high-risk elderly medical patients through delivery of a targeted care bundle. J Hosp Med. 2009;4(4):211-218.

7. Jack BW, Chetty VK, Anthony D, et al. A reengineered hospital discharge program to decrease rehospitalization: a randomized trial. Ann Intern Med. 2009;150(3):178-187.

8. Fitzgerald JF, Smith DM, Martin DK, Freedman JA, Katz BP. A case manager intervention to reduce readmissions. Arch Intern Med. 1994; 154(15):1721-1729.

9. Coleman EA, Smith JD, Frank JC, Min SJ, Parry C, Kramer AM. Preparing patients and caregivers to participate in care delivered across settings: the Care Transitions Intervention. I Am Geriatr Soc. 2004; 52(11):1817-1825

10. Forster AJ, Clark HD, Menard A, et al. Effect of a nurse team coordinator on outcomes for hospitalized medicine patients. Am J Med. 2005;118(10):1148-1153.

11. Arora VM, Prochaska ML, Farnan JM, et al. Problems after discharge and understanding of communication with their primary care physicians among hospitalized seniors: a mixed methods study. J Hosp Med. 2010;5(7):385-391.
12. Anderson GF, Steinberg EP. Hospital readmissions in the Medicare population. N Engl J Med. 1984;311(21):1349-1353.

13. Baker DW, Williams MV, Parker RM, Gazmararian JA, Nurss J. Development of a brief test to measure functional health literacy. Patient Educ Couns. 1999;38(1):33-42.

14. Lindquist LA, Pollack J, Go L, Jain N, Friesema E, Baker DW. Relationship of Health Literacy to Intentional and Unintentional NonAdherence of Hospital Discharge Medications. J Gen Intern Med. 2012;27(2):173-178.

15. Krumholz HM, Parent EM, Tu N, et al. Readmission after hospitalization for congestive heart failure among Medicare beneficiaries. Arch Intern Med. 1997;157(1):99-104.

16. Courtenay WH. College men's health: an overview and a call to action. I Am Coll Health. 1998;46(6):279-290.

17. Lorber J. Gender and the Social Construction of Illness. Thousand Oaks, CA: Sage; 1997.

18. Whitehead M. Inequalities in Health: The Black Report and the Health Divide. London, UK: Penguin; 1988.

19. Patten J, Watt I, Lewin RJP, Standford N. Decision making process in people with symptoms of acute myocardial infarction: qualitative study. BMJ. 2002;332:1006-1017.

20. Centers for Medicare and Medicaid Services. Transitional Care Management Services. Available at: http://www.cms.gov/Medicare/ Medicare-Fee-for-Service-Payment/PhysicianFeeSched/Downloads/FAQTCMS.pdf. Accessed June 28, 2013. 